\title{
The transition from symmetric to baroclinic instability in the Eady model
}

\author{
Megan A. Stamper ${ }^{1} \cdot$ John R. Taylor ${ }^{1}$ (i)
}

Received: 1 July 2016 / Accepted: 18 October 2016 / Published online: 19 November 2016

(C) The Author(s) 2016. This article is published with open access at Springerlink.com

\begin{abstract}
Here, we explore the transition from symmetric instability to ageostrophic baroclinic instability in the Eady model; an idealised representation of a submesoscale mixed layer front. We revisit the linear stability problem considered by Stone (J Atmos Sci, 23, 390-400, 1966), Stone (J Atmos Sci, 27, 721-726, 1970), Stone (J Atmos Sci, 29, 419-426, 1972) with a particular focus on threedimensional 'mixed modes' (which are neither purely symmetric or baroclinic) and find that these modes can have growth rates within just a few percent of the corresponding two-dimensional growth rate maximum. In addition, we perform very high resolution numerical simulations allowing an exploration of the transition from symmetric to baroclinic instability. Three-dimensional mixed modes represent the largest contribution to the turbulent kinetic energy during the transition period between symmetric and baroclinic instability. In each simulation, we see the development of sharp fronts with associated high rms vertical velocities of up to $30 \mathrm{~mm} \mathrm{~s}^{-1}$. Furthermore, we see significant
\end{abstract}

This article is part of the Topical Collection on the 48th International Liège Colloquium on Ocean Dynamics, Liège, Belgium, 23-27 May 2016

Responsible Editor: Alexander Barth

John R. Taylor

J.R.Taylor@damtp.cam.ac.uk

Megan A. Stamper

M.A.Stamper@damtp.cam.ac.uk

1 Department of Applied Mathematics and Theoretical Physics, Centre for Mathematical Sciences, Wilberforce Road, Cambridge, CB3 0WA, UK transfer of energy to small scales, demonstrated by timeintegrated mixing and energy dissipation by small-scale three-dimensional turbulence totalling about $30 \%$ of the initial kinetic energy in all cases.

Keywords Submesoscale Turbulence $\cdot$ Instability

\section{Introduction}

The mixed layer at the surface of the ocean plays an important role in the Earth's climate system. Water properties at the ocean surface influence the rate of exchange of heat, carbon, and other important tracers between the atmosphere and the ocean. Vertical motions and three-dimensional turbulence then exchange fluid between the mixed layer and the more strongly stratified waters beneath. Dynamical processes within the mixed layer influence the exchange of tracers between the atmosphere and deep ocean by modifying surface water properties, the mixed layer depth, and vertical fluxes at the mixed layer base.

Despite its name, the properties of the mixed layer are not uniform (e.g., Rudnick and Ferrari 1999). Fronts, defined here as regions with sharp density contrasts, are commonplace in the upper ocean. Fronts occur on a wide range of scales, from major frontal systems including western boundary currents and the Antarctic Circumpolar Current to small scale shelf break and tidal fronts. Fronts are hotspots for the formation and subduction of 'mode water' (Joyce et al. 2009) and biological productivity (Taylor and Ferrari 2011).

Ocean fronts are often close to thermal wind balance, but this equilibrium is unstable to a variety of submesoscale instabilities. Submesoscales are characterized by scales generally between 1-10 km and Rossby numbers of order unity. Submesocales play a major role in controlling the 
dynamics and structure of the upper ocean by enhancing the vertical exchange between the ocean surface and waters below, while re-stratifying the mixed layer. The resulting motion can have a strong influence on tracer transport and biological productivity (Lévy et al. 2012; Thomas et al. 2008).

Significant attention has been given to identifying various submesoscale processes and instabilities at mixed layer fronts, including ageostrophic baroclinic instability (Boccaletti et al. 2006), symmetric instability (Taylor and Ferrari 2009), anticyclonic ageostrophic instability (McWilliams et al. 2004), and submesoscale frontogensis (Shakespeare and Taylor 2013). However, the interactions between various submesoscale processes has received less attention. Here, we consider an idealized flow that is unstable to both symmetric and ageostrophic baroclinic instability and explore the interaction between these two submesoscale processes.

Symmetric instability can occur when the potential vorticity in a volume of fluid takes the opposite sign of the Coriolis parameter (Hoskins 1974), and the most unstable perturbations are independent of the along-front direction (Stone 1966). Symmetric instability can be described as a generalization of gravitational and inertial instability, (Haine and Marshall 1997; Thomas et al. 2013). See also (Cushman-Roisin and Beckers 2011) for a discussion of the relationship between these instabilities. Here, we will only consider cases that are stable to gravitational and inertial instability.

To study the interaction between symmetric instability, baroclinic instability, and turbulence, we use a very idealised representation of the ocean mixed layer, the Eady model, first described by Eady in 1949 as a prototype of quasi-geostrophic baroclinic instability (Eady 1949). Later, Stone $(1966,1970,1972)$ used this model to study ageostrophic instabilities. Stone's work formed the foundation for a parameterization of baroclinic instability in the mixed layer (so-called mixed layer instability, or MLI) (Fox-Kemper et al. 2008b). Variants of this model have been used in previous studies of symmetric and baroclinic instability (e.g., Molemaker et al. 2010; Taylor and Ferrari 2009; Arobone and Sarkar 2015).

The Eady model consists of a fluid with uniform potential vorticity between two rigid horizontal boundaries. The buoyancy is a linear function of $y$ and $z$ with $N^{2}=\partial b / \partial z$ and $M^{2}=\partial b / \partial y$, where $N$ is the buoyancy frequency and $M$ is its horizontal analogue. To simplify the discussion, we assume that changes in buoyancy are dominated by temperature changes, with a linear equation of state. The velocity is aligned with the $x$-direction and is in thermal wind balance such that $d u_{G} / d z=-M^{2} / f$. As noted by Stone (1966), the inviscid Eady model is characterized by a single non-dimensional parameter, the balanced Richardson number,

$R i=\frac{N^{2} f^{2}}{M^{4}}$,

which is also the gradient Richardson number associated with the initial state,

$\operatorname{Ri}_{g}=\frac{N^{2}}{\left(\frac{d u_{G}}{d z}\right)^{2}}=\frac{N^{2} H^{2}}{U_{G}^{2}}=\mathrm{Ri}$,

where $U_{G}=\frac{M^{2} H}{f}$ is the characteristic velocity scale.

Stone (1966) used asymptotic expansions to analyse the linear stability of normal mode perturbations and found that symmetric instability occurs only when $\mathrm{Ri}<1$. Symmetric modes, or those independent of the along-front direction, grow fastest when $\mathrm{Ri}<0.95 .{ }^{1}$ For the basic state of the Eady problem, $\mathrm{Ri}<1$ corresponds to $f q<0$, where $q$ is the Ertel potential vorticity (PV). In Eady's original analysis, which only admitted geostrophic perturbations, baroclinic instability occurs only when Ri > 0.84 (Eady 1949). By contrast, Stone's analysis permits ageostrophic perturbations and admits growing baroclinic modes, or those independent of the across-front direction, for all Ri. As a result, there is a range of Ri for which baroclinic and symmetric instability may occur simultaneously. In addition, 'mixed modes' can develop with both $x$ and $y$ dependence. To date, relatively little attention has been paid to the growth rate and properties of mixed modes, but we find that they are important in describing the transition from symmetric to baroclinic instability.

The plan of the paper is as follows. In Section 2, we revisit the linear stability problem considered by Stone $(1966,1970,1972)$ with a particular emphasis on the threedimensional 'mixed modes' which cannot be purely categorized as symmetric or baroclinic. In Section 3, we use very high resolution numerical simulations of the Eady flow for several different Richardson numbers and explore the transition from symmetric to baroclinic instability, the appearance of mixed modes, the mixing and energy dissipation by small-scale three-dimensional turbulence, and the development of sharp fronts and the associated vertical velocities.

\footnotetext{
${ }^{1}$ In fact, Stone claimed that symmetric modes were fastest growing in the slightly smaller interval $0.25<\mathrm{Ri}<0.95$, with Kelvin-Helmholtz instability taking over in the lower range. However, Vanneste (1993) showed that this lower bound was erroneous and that the fastest growth rates are associated with symmetric instability in the full range $\mathrm{Ri}<$ 0.95 .
} 


\section{Linear stability analysis}

The Eady model consists of an initial state with linear vertical and meridional buoyancy gradients, denoted $\bar{b}(y, z)=$ $M^{2} y+N^{2} z$, in hydrostatic and geostrophic balance with a vertically-sheared along-front thermal wind, $u_{G}(z)$, such that

$\frac{d u_{G}}{d z}=-\frac{1}{f} \frac{\partial \bar{b}}{\partial y}$,

where $f$ is the Coriolis frequency. We split the velocity and buoyancy fields into a 'background' and perturbation part,

$\mathbf{u}=u_{G}(z) \mathbf{i}+\mathbf{u}^{\prime}(x, y, z, t)$,

$b=\bar{b}(y, z)+b^{\prime}(x, y, z, t)$.

Horizontal directions are taken to be periodic in the perturbation quantities, $b^{\prime}$ and $\mathbf{u}^{\prime}$.

The linear stability of normal mode perturbations to this basic state follows using perturbations of the form $u=$ $\hat{u}(z) e^{\sigma t+i(k x+\ell y)}$, where $\sigma$ is the complex growth rate, $k$ is the $x$-direction wavenumber, and $\ell$ is the $y$-direction wavenumber. The system will be unstable when $\sigma$ has a positive real part. The wavenumbers and growth rates $k, \ell$, and $\sigma$ are dimensionless, made non-dimensional from the dimensional variables $\breve{k}, \check{\ell}$, and $\check{\sigma}$ by taking

$$
\begin{aligned}
(k, \ell) & =\frac{f}{U_{G}}(\check{k}, \check{\ell}), \\
\sigma & =f \check{\sigma} .
\end{aligned}
$$

Here, we will define the terms 'baroclinic' and 'symmetric' instability to represent two limiting cases of the perturbation analysis. We will define symmetric instability as perturbations which are independent of the along-front direction, i.e., with along-front wavenumber $k=0$. Similarly, baroclinic instability is defined as perturbations which are independent of the cross-front direction, i.e., with crossfront wavenumber $\ell=0 .^{2}$ Finally, we define 'mixed' modes as those perturbations with both $x$ and $y$ dependence, i.e., with $k \neq 0$ and $\ell \neq 0$. Following Stone (1966), when plotting in $(k, \ell)$ space, we will refer to the $k$-axis as the baroclinic axis and the $\ell$-axis as the symmetric axis.

Using the normal mode form for linear perturbations to the basic state and eliminating variables results in

\footnotetext{
${ }^{2}$ It is perhaps worth noting that often the term 'baroclinic instability' encompasses all of the above instabilities found to arise in a rotating, stratified fluid subject to a horizontal temperature gradient, while here we restrict this definition to the purely two-dimensional instability with $\ell=0$. This is done partly to remain consistent with Stone's work but also as it allows for ease in distinguishing between the two 2-dimensional instabilities of this system.
}

an eigenvalue problem for the vertical velocity, $w=$ $\hat{w}(z) e^{\sigma t+i(k x+\ell y)}$, as first expressed by Eady (1949):

$$
\begin{aligned}
\left(1-(i \sigma+k z)^{2}\right) \frac{d^{2} \hat{w}}{d z^{2}} & -\left(\frac{2 k}{i \sigma+k z}-2 i \ell\right) \frac{d \hat{w}}{d z} \\
& -\left(\operatorname{Ri}\left(k^{2}+\ell^{2}\right)+\frac{2 i k \ell}{i \sigma+k z}\right) \hat{w}=0 .
\end{aligned}
$$

Stone (1966) solved this eigenvalue problem using an asymptotic analysis for small $k$ or small $\ell$.

We proceed instead by discretising Eq. 2 using secondorder centered finite differences. Then, for each value of $\mathrm{Ri}$, we step through spectral space using a shooting method requiring linear extrapolation of the growth rate at each wavenumber to satisfy the rigid lid boundary condition at $z=H$. This approach is similar to the method used in Stone (1970) although we solve Eq. 2 directly, rather than the algebraically simplified version solved by Stone; equation (2.6) from (Stone 1970). A full description of the numerical method can be found in Appendix A.

The results of this numerical linear stability analysis indicate a smooth transition from a maximum growth rate on the symmetric axis $(k=0)$ for low Ri to a maximum growth rate on the baroclinic axis $(\ell=0)$ for higher Ri. Figure 1 shows the growth rate as a function of $k$ and $\ell$ for $\mathrm{Ri}=0.25,0.5,0.75$, and 1 . Although the most unstable mode always has either $k=0$ or $\ell=0$, there are large regions of mixed modes $(k, \ell \neq 0)$ with non-negligible growth rates. For example, at $\mathrm{Ri}=1$, the most unstable mode is on the baroclinic axis $(\ell=0)$ with a growth rate of $\sigma=0.23$ and wavenumber $k=1.19$. For comparison, the mixed mode with equal wavenumbers in the $x$ and $y$ directions, $k=\ell=1.19$ has a growth rate $\sigma=0.22,96 \%$ of the maximum.

From the linearised governing equations, we can obtain a domain averaged equation for the evolution of perturbation kinetic energy,

$$
\begin{aligned}
& \frac{1}{2} \frac{\partial}{\partial t} \iiint\left(\tilde{u} \tilde{u}^{*}+\tilde{v} \tilde{v}^{*}+\tilde{w} \tilde{w}^{*}\right) d k d \ell d z \\
& =\iiint\left(-\tilde{u} \tilde{w}^{*} \frac{d u_{G}}{d z}+\tilde{b} \tilde{w}^{*}\right) d k d \ell d z
\end{aligned}
$$

where $(\tilde{\cdot})$ denotes Fourier coefficients such that $\left(u^{\prime}, v^{\prime}, w^{\prime}\right)=$ $\iint(\tilde{u}, \tilde{v}, \tilde{w}) e^{i(k x+\ell y)} d k d \ell$ with $(\tilde{u}, \tilde{v}, \tilde{w})=(\hat{u}(z), \hat{v}(z)$, $\hat{w}(z)) e^{\sigma t}$, and stars denote complex conjugates. The two terms on the right hand side represent geostrophic shear production and buoyancy flux, respectively, relating to transfer of kinetic energy from the kinetic and potential energy budgets of the background state in thermal wind balance.

After solving Eq. 2 for $\hat{w}$ and $\sigma$, the linearised governing equations can be used to obtain $\hat{u}$ and $\hat{b}$. Thus, we can calculate the resulting geostrophic shear production and buoyancy flux terms throughout $(k, \ell)$ space from the 
Fig. 1 Dimensionless growth rate, $\sigma$, as a function of along-front $(k)$ and cross-front $(\ell)$ wavenumbers for several Richardson numbers a $\mathrm{Ri}=0.25, \mathbf{b} \mathrm{Ri}=0.5$, $\mathbf{c} \mathrm{Ri}=0.75$, and $\mathbf{d} \mathrm{Ri}=1$. Growth rates are nondimensionalised using Coriolis frequency $f$ and lengths with $U_{G} / f$. In each case, the black dot on the baroclinic axis $(\ell=0)$ indicates the smallest wavenumber admitted by our choice of domain width for the simulations in Section 3 and was picked to ensure a growth rate of at least $90 \%$ of the predicted baroclinic maximum in all cases. The asterisk in Fig. 1a shows the mixed mode chosen for Fig. 8c (a)

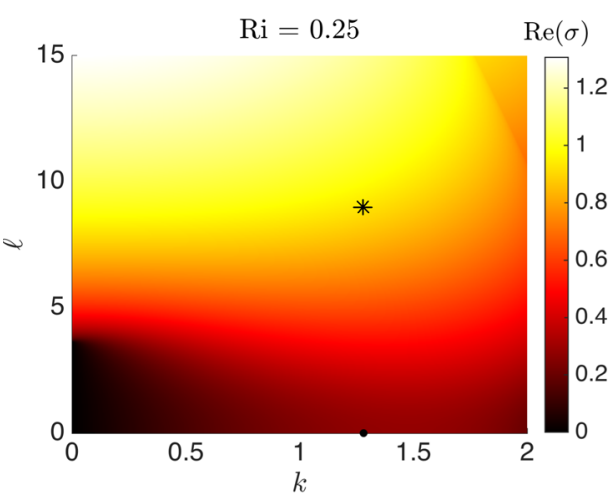

(c)

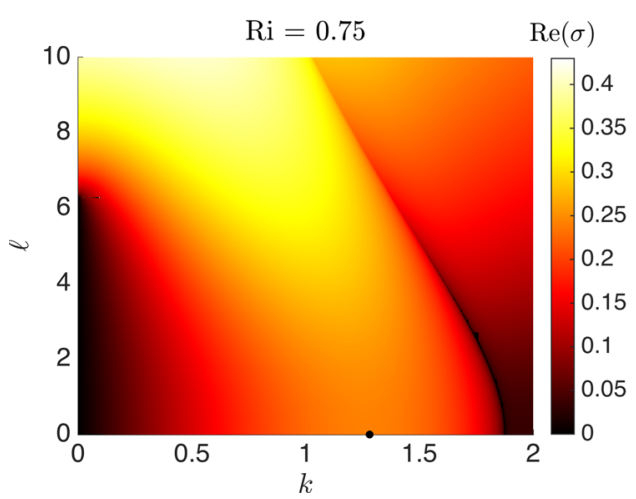

(b)

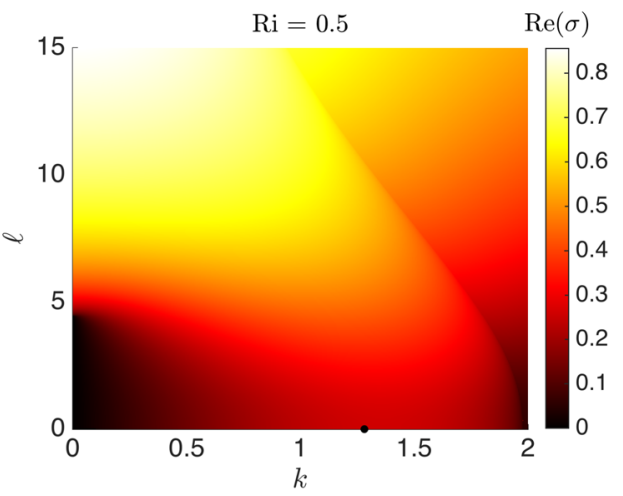

(d)

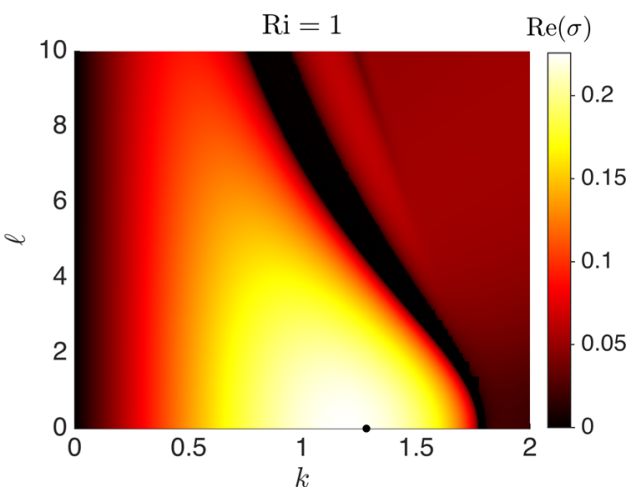

linear stability analysis. Figure 2 a shows the ratio of the geostrophic shear production and buoyancy flux,

$\gamma(k, \ell)=\frac{\int-\hat{u} \hat{w}^{*} \frac{d u_{G}}{d z} d z}{\int \hat{b} \hat{w}^{*} d z}(k, \ell)$,

in the case $\mathrm{Ri}=0.5$. The geostrophic shear production is small in comparison to the buoyancy flux along the baroclinic axis $(\ell=0)$ but becomes large on the symmetric axis $(k=0)$ in the limit with $\ell \gg 1$. In Fig. $2 \mathrm{~b}$, we show $\gamma$ for a selection of mixed modes with $\mathrm{Ri}=0.5$, i.e., modes with $k \neq 0$ and $\ell \neq 0$. The choice of mixed modes displayed is indicated by the white dashed line in Fig. 2a and represents the along-front wavenumber, $\ell$, corresponding to the maximum growth rate for fixed along-front wavenumber, $k$. The cross-front wavenumber of this mixed mode local maximum is denoted $\ell=\ell_{m m}$.

As we move from the baroclinic axis $(\ell=0)$ towards the symmetric axis $(k=0)$ along this line of mixed modes, we observe that the ratio of geostrophic shear production
Fig. 2 Plot a shows $\gamma(k, \ell)$ for $\mathrm{Ri}=0.5$; $\mathbf{b}$ shows $\gamma$ for mixed modes with $\ell=\ell_{\mathrm{mm}}$ where $\ell_{\mathrm{mm}}$ is defined as the cross-front wavenumber defined by the local maximum in $\operatorname{Re}(\sigma)$ indicated by the white dashed line in (a) (a)

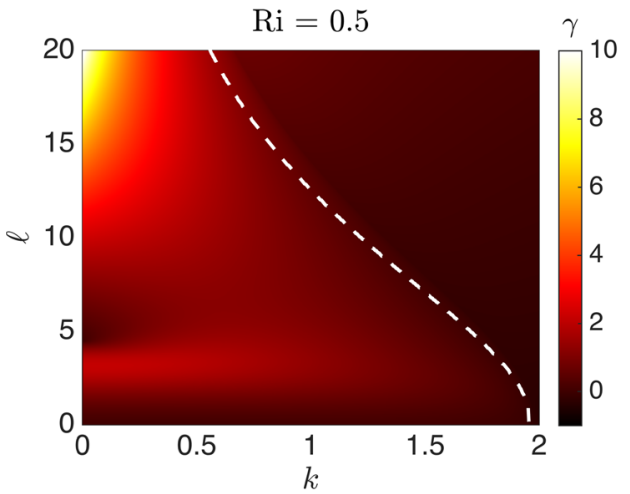

(b)

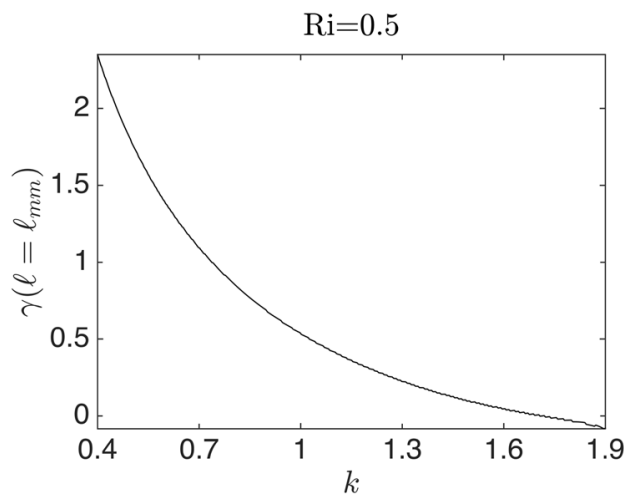


against buoyancy flux transitions gradually from $\gamma \ll 1$ near the baroclinic axis $(\ell=0)$, consistent with the expected behaviour for baroclinic modes, to $\gamma>1$ near the symmetric axis $(k=0)$. This implies that growing modes close to the baroclinic axis $(\ell=0)$, with $\ell<1$, can be thought of as 'baroclinic-like', with energetics similar in nature to baroclinic instability. Conversely, modes very close to the symmetric axis $(k=0)$, and with $\ell \gg 1$, have $\gamma>1$ and are more like symmetric modes in the limit $\ell \gg 1$. However, there are also a wide range of mixed modes far from these limits in which $\gamma=O(1)$, indicating that energy extraction from the kinetic energy and potential energy budgets are of comparable importance. For this particular set of mixed modes, $\gamma=1$ occurs where $k=0.6$ and $\ell=19.1$. Although the geostrophic shear production and buoyancy flux are comparable, this mode is nearly aligned with the along-front (symmetric) direction with $\ell \gg k$.

\section{Numerical simulations}

\subsection{Setup}

While linear stability analysis is useful for identifying and characterising instabilities, it cannot address the nonlinear evolution that occurs during later stages, nor describe possible interactions between symmetric, baroclinic, and mixed modes. In cases with $\mathrm{Ri}<0.95$, we expect that symmetric instability will grow fastest, based on the higher growth rates predicted by the linear theory, as discussed in Section 2. Since symmetric instability acts to restratify and can go unstable to a secondary Kelvin-Helmholtz instability (Taylor and Ferrari 2009), it may result in a modification of the background flow upon which more slowly growing baroclinic modes evolve. In addition, the growth and nonlinear saturation of primary instabilities is likely to induce heightened dissipation and small-scale mixing which cannot be quantified by linear theory.

To examine these issues, we perform high resolution numerical simulations of the Boussinesq, non-hydrostatic governing equations using 'Diablo', an open-source CFD solver (Taylor 2008). This solver uses a psuedo-spectral method in the horizontal, with periodic boundary conditions for $\mathbf{u}^{\prime}$ and $b^{\prime}$, and second-order finite differences in the vertical, where we impose no normal flow conditions $w^{\prime}=0$ and $\frac{\partial u^{\prime}}{\partial z}=\frac{\partial v^{\prime}}{\partial z}=\frac{\partial b^{\prime}}{\partial z}=0$ at $z=-H, 0$. Time stepping is implemented using two schemes; an implicit Crank-Nicolson scheme for viscous terms and an explicit low-storage, thirdorder Runga-Kutta scheme for other terms.

We use an isotropic viscosity, $v=6 \times 10^{-4} \mathrm{~m}^{2} \mathrm{~s}^{-1}$ and take the Prandtl number, $\operatorname{Pr}=\frac{\nu}{\kappa}=1, \kappa$ being the thermal diffusivity. These simulations can be considered as a low
Reynolds number analysis of the full scale ocean setting or $v$ can be viewed as an eddy viscosity associated with unresolved turbulence in the mixed layer (the choice of Prandtl number is consistent with this latter interpretation).

Each simulation is performed on a domain of aspect ratio $\alpha=\frac{L_{x}}{H}=50$, where $L_{x}$ and $H$ are the alongfront horizontal and vertical dimensions, respectively. An initial Richardson number is chosen for each simulation, $\mathrm{Ri}_{0}=N^{2} f^{2} / M^{4}$, imposed by initialising with a uniform vertical and lateral density gradient. Throughout the simulations, $M^{2}$, the horizontal density gradient associated with the background state, is held constant by application of periodic boundary conditions on $b^{\prime}$. The velocity field is initialised in thermal wind balance with an additional white noise perturbation of amplitude $10^{-6} \mathrm{~ms}^{-1}$.

Here we take $L_{x}=L_{y}=2.5 \mathrm{~km}$ and $H=50 \mathrm{~m}$ as horizontal and vertical dimensions. The choice of $L_{x}$ and $L_{y}$ is set to ensure computational efficiency whilst still being large enough to ensure that the growth rate of the fastest growing baroclinic mode in all simulations, denoted by the black dot in each panel of Fig. 1, is within $10 \%$ of the theoretical maximum. A fine, regularly-spaced grid, with 64 vertical points, and $1024^{2}$ horizontal points, the maximum possible given current computational resources, gives grid-spacing of $0.8 \mathrm{~m}$ and $2.4 \mathrm{~m}$ in the vertical and horizontal directions, respectively. The initial gradient Richardson number, $\mathrm{Ri}_{0}$, is set by choosing the parameters $M^{2}=1 \times 10^{-7} \mathrm{~s}^{-2}, f=10^{-4} \mathrm{~s}^{-1}$, and $N^{2}=$ $\mathrm{Ri}_{0} \times 10^{-6} \mathrm{~s}^{-2}$.

(a)

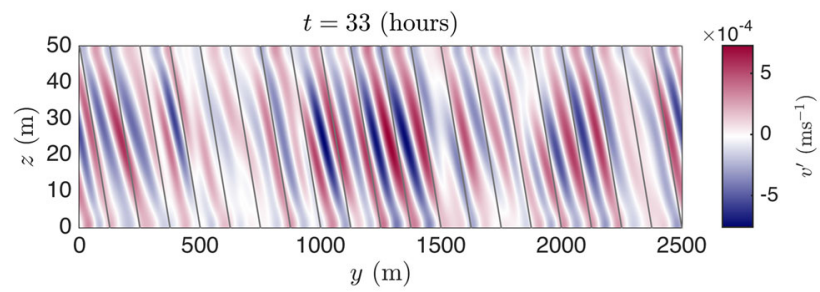

(b)

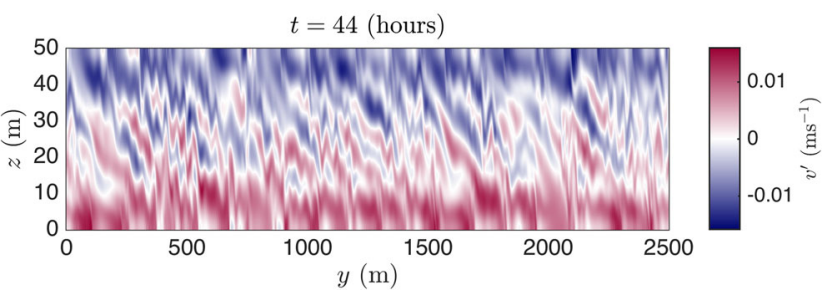

Fig. 3 From the $\mathrm{Ri}_{0}=0.25$ simulation we see $\mathbf{a}$ appearance of symmetric instability convection cells in the cross-front velocity field at $33 \mathrm{~h}$, grey lines indicate the isopycnal slope for comparison; b secondary Kelvin-Helmholtz instability acting on the shear layers between symmetric instability convection cells at $44 \mathrm{~h}$ 
(a)

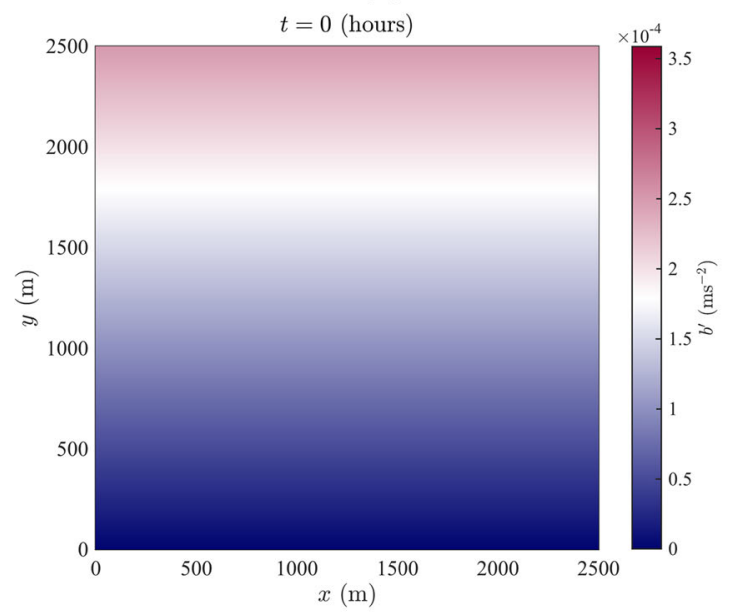

(c)

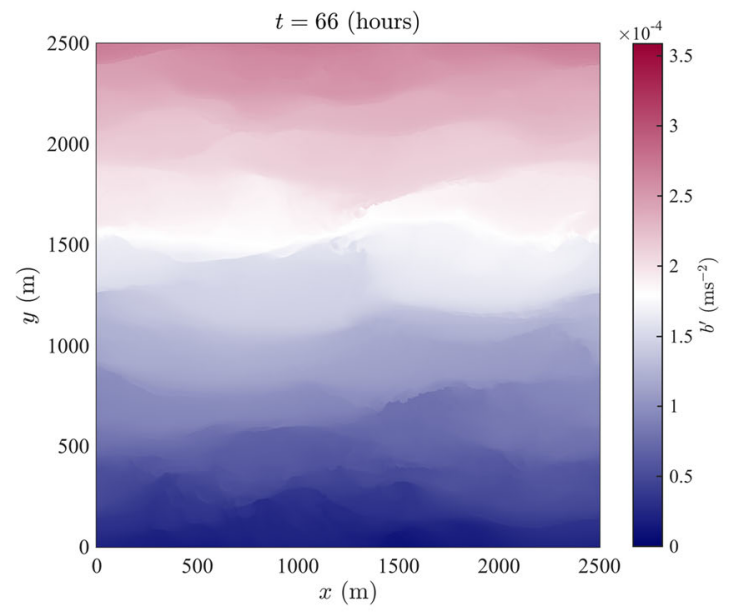

(e)

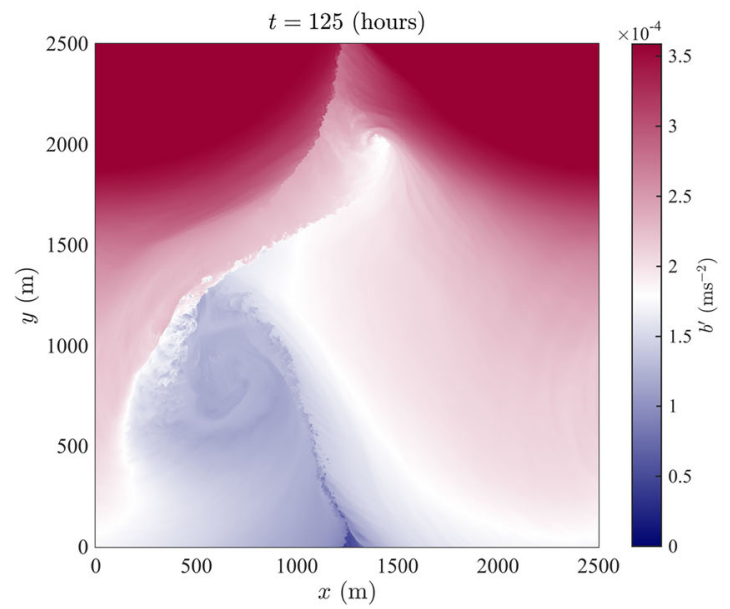

(b)

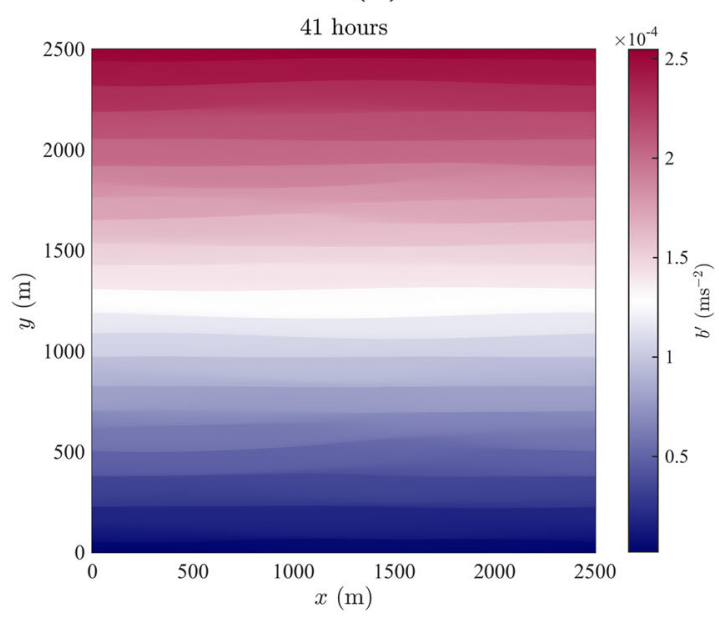

(d)

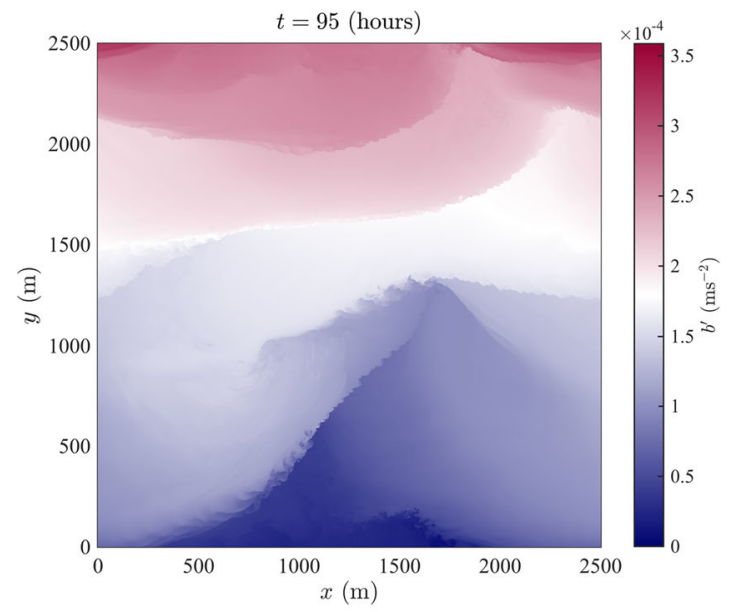

(f)

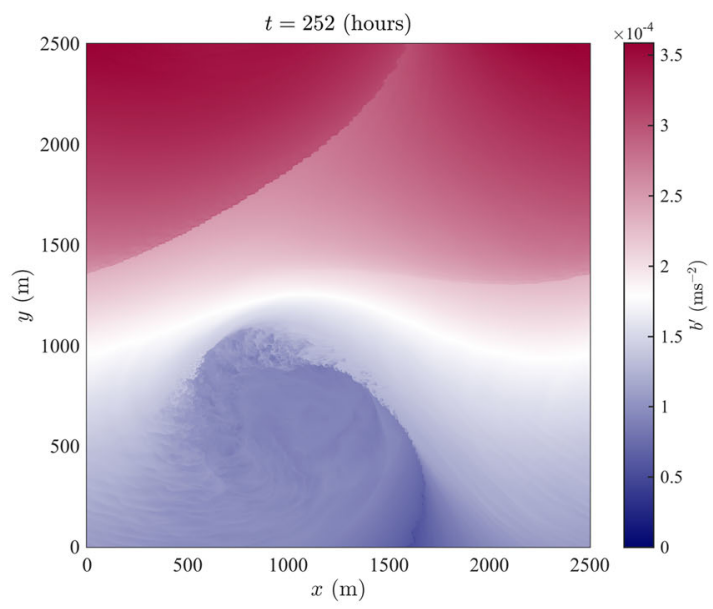

Fig. 4 The evolution of surface buoyancy at various times in the $\mathrm{Ri}=0.25$ simulation 


\subsection{General description}

We compare the results of four simulations with initial Richardson numbers, $\mathrm{Ri}_{0}=0.25,0.5,0.75$, and 1. Simulations with $\mathrm{Ri}_{0}<1$ are unstable to symmetric instability and baroclinic instability, while the $\mathrm{Ri}_{0}=1$ case is unstable to baroclinic instability only. Initially, in this section, we will focus on a description of the $\mathrm{Ri}_{0}=0.25$ case with both symmetric and baroclinic modes having the largest predicted linear growth rates of all simulations.

The first feature seen in the $\mathrm{Ri}_{0}=0.25$ simulation is a growing symmetric mode. Visualisations of the cross-front velocity field in the $\mathrm{Ri}_{0}=0.25$ simulation are shown in Fig. 3. A snapshot of the flow after $33 \mathrm{~h}$ (Fig. 3a) shows the development of symmetric instability in the form of velocity cells nearly aligned with isopycnals. After about $40 \mathrm{~h}$, this symmetric mode begins to saturate and, as the magnitude of local shear grows at the edges of the symmetric instability cells, we soon see the appearance of secondary, small-scale Kelvin-Helmholtz (KH) instabilities, as shown in Fig. $3 b$. This figure also highlights the development of cross-front flow. Negative cross-front velocity at the surface and positive cross-front velocity at the bottom acts to restratify the front as in previous work (Taylor and Ferrari 2009).

In addition to the along-isopycnal cells associated with symmetric instability seen in the cross-front velocity, we also observe the emergence of a series of regularly spaced fronts, or 'frontal bands', in the density field at the surface and base of the mixed layer (see Fig. 4b). The initial wavelength of these bands is the same as the wavelength of the symmetric instability cells, but their wavelength increases over time (see Figs. $4 \mathrm{c}, \mathrm{d}$ ). The increase in the scale of the frontal bands occurs as saturation of the fastest-growing, smallest-scale symmetric mode leaves behind larger-scale symmetric and mixed modes growing in its wake. The increase in wavelength of these bands continues from their first appearance until the Richardson number, Ri $=\bar{N}^{2} f^{2} / M^{4}$, where $\bar{N}$ is the domainaverage buoyancy frequency, and $M^{2}$ is the constant background buoyancy gradient, reaches $\mathrm{Ri} \approx 1$ (see Fig. 6) at which point symmetric instability growth is arrested and baroclinic modes take over as the fastest growing instability.

The inclusion of the third dimension in our simulation captures an interesting feature of this surface frontal band formation process; we see a further transition to smallscale secondary instability at edges of the frontal bands (see Fig. 4c). We believe that this horizontal secondary instability is caused by continued frontogenesis which, acting to strengthen the fronts, results in along-front velocity shear (see Fig. 5a) allowing the growth of horizontal shear instabilities. After $66 \mathrm{~h}$, the development of large scale variations in $x$ indicates the presence of growing baroclinic and mixed modes (see Fig. 4c).

Once the background flow has reached a point of stability with regard to symmetric instability, i.e., reached a state with $\mathrm{Ri} \geq 1$, growth in TKE is then predominantly from baroclinic and mixed mode instabilities, which we can (a)

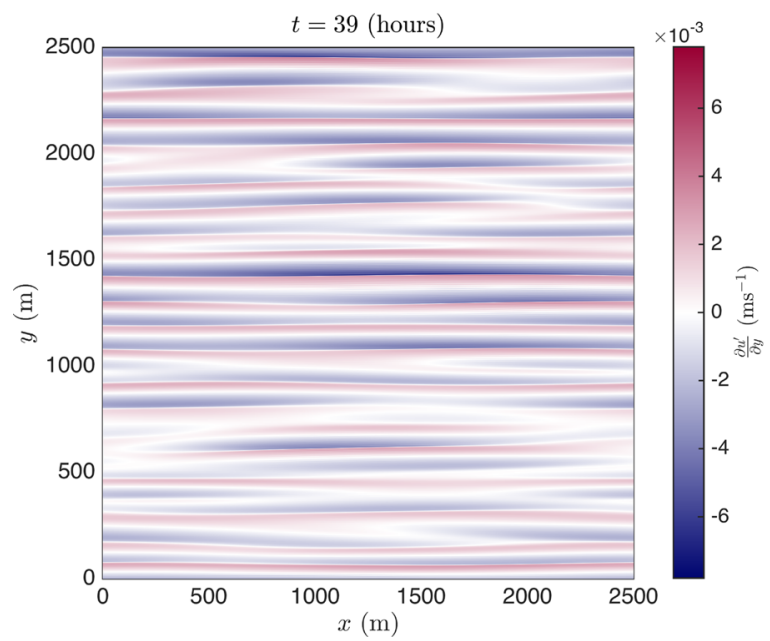

(b)

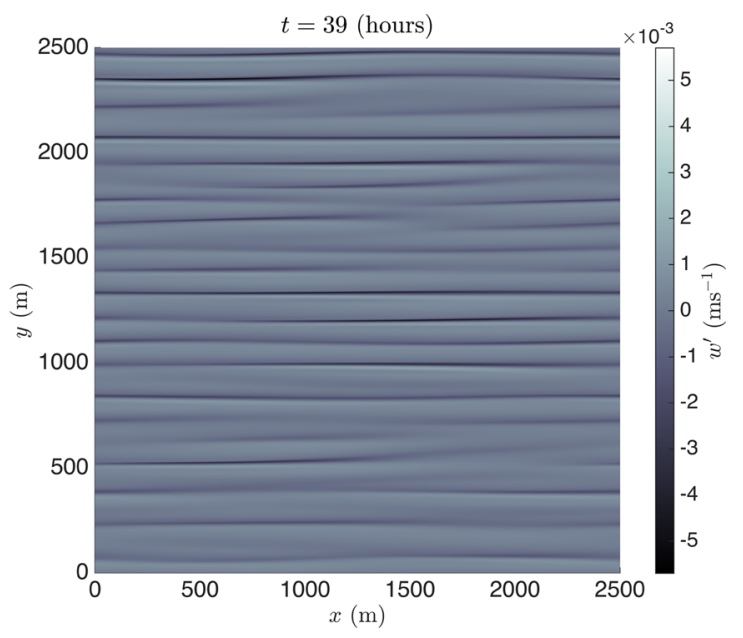

Fig. 5 a Along front shear at the surface and $\mathbf{b}$ vertical velocity, $w^{\prime}$, at $5 \mathrm{~m}$ depth from the $\mathrm{Ri}_{0}=0.25$ simulation at $39 \mathrm{~h}$. This time corresponds to the first local maxima of $w_{\text {rms }}$ as shown in Fig. 14 
see deforming the surface buoyancy field as in Fig. 4d. A final transition to turbulence can then be seen towards the later stages of each simulation where, after $95 \mathrm{~h}$, we see the appearance of fine scale structures along the fronts and filaments distorted by a developing large scale baroclinic mode. Eventually, the flow develops into a state with a sharp frontal band wrapped around a coherent submesoscale eddy.

\subsection{Transition from symmetric to baroclinic instability}

In this section, we will examine the nonlinear transition from symmetric to baroclinic instability. We will focus on how the earlier development of symmetric instability in simulations with $\mathrm{Ri}_{0}<1$ impacts the later instability characteristics, restratification, and energetics.

There is an overall increase in Ri over time in each simulation (see Fig. 6). Since $M^{2}$ is held constant, this signals increasing stability of the flow with shallowing isopycnal slopes and an increase in $N^{2}$. Further, it appears that $\mathrm{Ri}$ begins to attain an equilibrium value in the latter stages of each simulation. Adjustment of Ri occurs more quickly in low $\mathrm{Ri}_{0}$ cases, with both maximum and equilibrium values of Ri being obtained more quickly. Finally, oscillations present in this figure for all simulations appear to be nearinertial, with timescales close to the inertial period $\frac{2 \pi}{f} \approx$ $17.5 \mathrm{~h}$.

If we use Reynold's decomposition on deviations from the thermal wind component of the velocity, $u_{G}=\frac{M^{2} z}{f}$, to split $\mathbf{u}$ into a mean part (a domain average in this case, denoted by the bar) and fluctuations from the mean

$\mathbf{u}=u_{G}(z) \hat{\mathbf{i}}+\overline{\mathbf{u}}+\mathbf{u}^{\prime}$

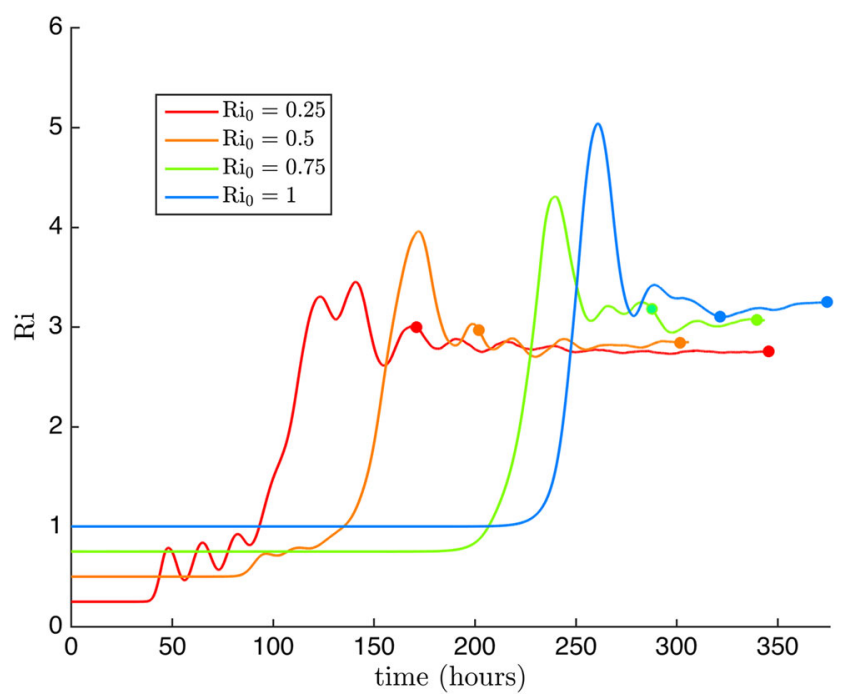

Fig. 6 Temporal evolution of Ri throughout each of our 3D simulations. Closed circles indicate the mean end-time for total integrated statistics as discussed in Section 3.4 we obtain an equation for the evolution of turbulent kinetic energy (TKE), TKE $\equiv \frac{1}{2} \overline{\left(u^{\prime 2}+v^{\prime 2}+w^{\prime 2}\right)}$,

$\frac{\partial(T K E)}{\partial t}=P+B-\varepsilon+T$

with the first three components on the right hand side being

$P=\underbrace{-\overline{u^{\prime} w^{\prime}} \frac{\overline{d u_{G}}}{d z}}_{\text {GSP }}-\underbrace{\overline{\mathbf{u}^{\prime} w^{\prime}} \cdot \frac{\partial \overline{\mathbf{u}}}{\partial z}}_{\text {AGSP }}$,

the geostrophic shear production (GSP) and ageostrophic shear production (AGSP), respectively, $B=\overline{b^{\prime} w^{\prime}}$, the buoyancy flux and

$\varepsilon=2 v \overline{s_{i j} s_{i j}}$ with $s_{i j}=\frac{1}{2}\left(\frac{\partial u_{i}^{\prime}}{\partial x_{j}}+\frac{\partial u_{j}^{\prime}}{\partial x_{i}}\right)$,

the viscous dissipation of kinetic energy. The final term, $T$, denotes transport and is identically zero due to periodicity, rigid lid and free-slip boundary conditions.

In each case, the TKE increases with time until saturating at a nearly constant value (Fig. 7). Interestingly, the saturated levels of TKE are very similar in all cases. For each simulation, we pick out the first and last periods of exponential growth, indicated by circles and triangles, respectively, in Fig. 7. For the simulation with $\mathrm{Ri}=0.75$, there is some uncertainty in this choice given that the change in gradient is small. The first period of growth, indicated by circles, is associated with faster-growing symmetric instability, verified by examining snapshots of the flow as in Fig. 3. Symmetric instability eventually saturates, as seen at around $40 \mathrm{~h}$ in the $\mathrm{Ri}_{0}=0.25$ case, and then a transition to a second period of exponential growth occurs, indicated by

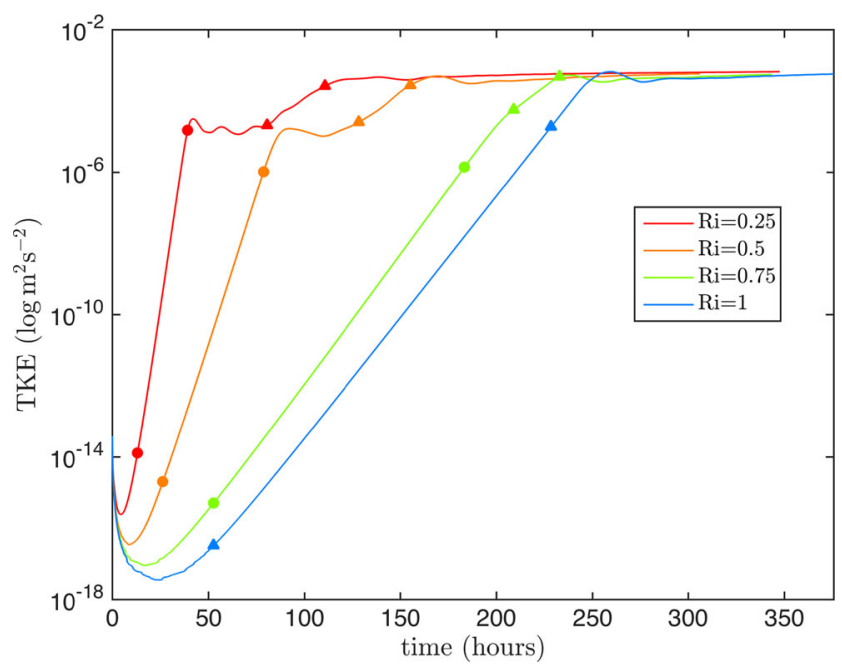

Fig. 7 Evolution of the turbulent kinetic energy for each simulation. Circles denote the time window used for calculation of symmetric growth rates, while triangles give the time window used for baroclinic growth rates 
triangles. In the $\mathrm{Ri}_{0}=1$ case, there is just a single period of exponential growth owing to the absence of symmetric instability when $\mathrm{Ri} \geq 1$. In simulations with smaller $\mathrm{Ri}_{0}$, the TKE attains both its peak and saturated values sooner.

We can quantitatively compare the linear predictions of Section 2 and the observed growth of the mean TKE by examining spectral decompositions of the TKE. The TKE contribution from symmetric modes is given by

$\operatorname{TKE}(k=0)=\left.\frac{1}{H} \iint\left(\tilde{u} \tilde{u}^{*}+\tilde{v} \tilde{v}^{*}+\tilde{w} \tilde{w}^{*}\right)\right|_{k=0} d \ell d z$.

The contribution from baroclinic modes is similar with $k$ and $\ell$ transposed, while for mixed modes it is

$\operatorname{TKE}(k \neq 0, \ell \neq 0)=\frac{1}{H} \iint_{k \neq 0, \ell \neq 0}\left(\tilde{u} \tilde{u}^{*}+\tilde{v} \tilde{v}^{*}+\tilde{w} \tilde{w}^{*}\right) d k d \ell d z$.
Figure 8 shows a comparison of predicted and computed growth rates for symmetric modes, baroclinic modes, and one choice of mixed mode. The diagnosed growth associated with symmetric modes agree well with Stone's analytic predictions in the limit $\ell \rightarrow \infty$ (solid line). The agreement is improved further by including viscosity in the linear prediction as in (Taylor and Ferrari 2009) (dashed line). The diagnosed growth rate associated with mixed modes also agrees well in the numerical simulations and linear theory. Figure $8 \mathrm{c}$ shows one example with $k=1.28$ and $\ell=8.98$. This mode is indicated by an asterisk in Fig. 1a.

However, looking at Fig. 8b, it seems that for all simulations with $\mathrm{Ri}_{0}<1$, the computed baroclinic growth rates during the second period of exponential TKE growth (open circles) deviate substantially from analytic predictions based on the initial Richardson number (solid line). (a)

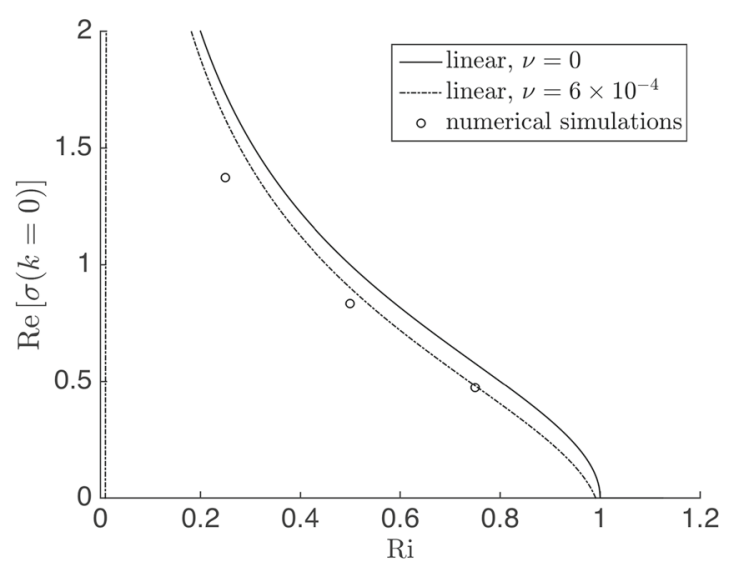

(b)

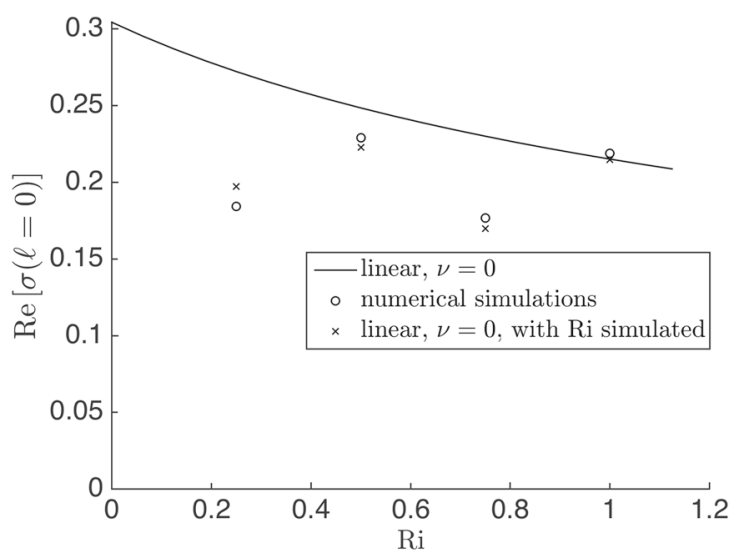

(c)

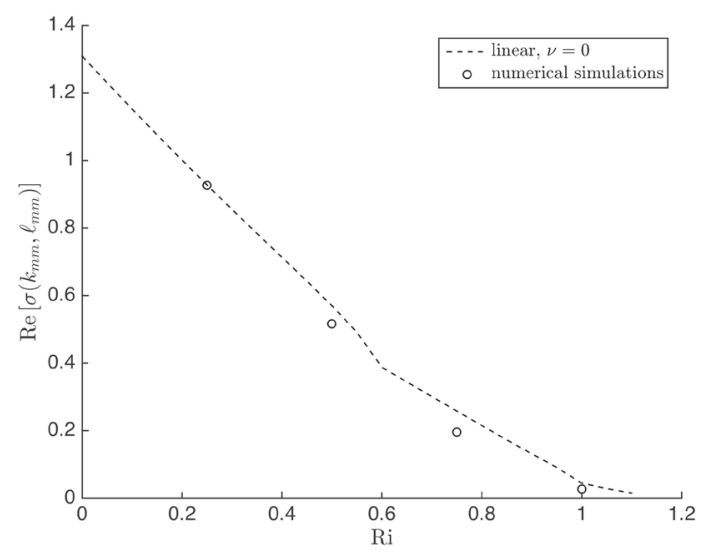

numerical simulations (open circles) and Stone's analytic baroclinic growth rate prediction calculated using the simulated mean Ri throughout the same period (crosses); $\mathbf{c}$ a comparison between the growth rate of a mixed mode with $k_{\mathrm{mm}}=1.28$ and $\ell_{\mathrm{mm}}=8.98$ throughout each simulation (open circles) with that predicted by our linear stability analysis (dashed line) 


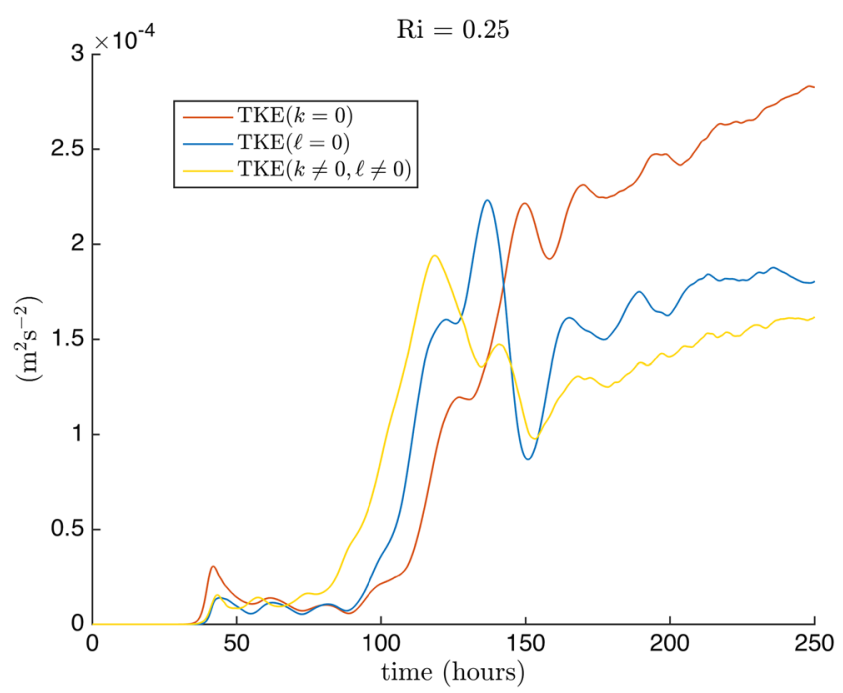

Fig. 9 A comparison of TKE split into contributions from symmetric (red), mixed (yellow), and baroclinic (blue) modes for the $\mathrm{Ri}_{0}=0.25$ simulation

This occurs as a result of the growth of faster non-baroclinic instabilities at early times which, both directly and via secondary instabilities, result in a modification of the mean stratification. If we take account of this effect by predicting linear growth rates using the mean Richardson number during this later linear growth period as defined in Fig. 7 (trianges) rather than the initial $\mathrm{Ri}_{0}$, we see substantially closer agreement between predicted and calculated values. This implies that the variation of the background state by faster growing symmetric and mixed modes has a substantial effect on the growth rate of slower growing baroclinic modes.
Figure 9 shows the temporal evolution of the decomposition of TKE into symmetric, baroclinic, and mixed modes in the $\mathrm{Ri}_{0}=0.25$ case. We see that symmetric modes give the most significant contribution to the TKE by about $50 \mathrm{~h}$. Then mixed modes contribute most between 75 and $125 \mathrm{~h}$, with baroclinic modes taking over between 125 and $150 \mathrm{~h}$. All three components are significant at late times, reflecting a three-dimensional eddying state.

The volume-averaged geostrophic shear production and buoyancy flux are shown for each case in Fig. 10. In the cases, with $\mathrm{Ri}_{0}=0.25$ and $\mathrm{Ri}_{0}=0.5$, the geostrophic shear production dominates at early times associated with symmetric instability. In the later stages of the simulations, where from Fig. 6, we can see that we are reaching states with $\mathrm{Ri}>1$, we begin to see large positive buoyancy fluxes, consistent with baroclinic instability.

Given that the geostrophic shear production and buoyancy flux peak in different phases of the evolution, we can examine their relative net contribution to the TKE throughout each simulation by integrating in time. For these integrals, we choose a cutoff time, $T$, to be where each simulation appears to first reach its final equilibrium state and variations in $\mathrm{Ri}$ are small. More precisely, $T$ is defined as the first time when the variance of Ri from time $T$ until the end time of the simulation, satisfies

$\operatorname{Var}\left(\left.\operatorname{Ri}\right|_{t=T} ^{t=t_{\text {end }}}\right)<\epsilon$.

We considered a range of choices, $2 \times 10^{-6}<\epsilon<$ $2 \times 10^{-3}$, to ensure observed energetic trends are independent of this arbitrary choice. We set $\epsilon$ to give an averaging
Fig. 10 A comparison of geostrophic shear production and buoyancy flux throughout each simulation (top and bottom, respectively)
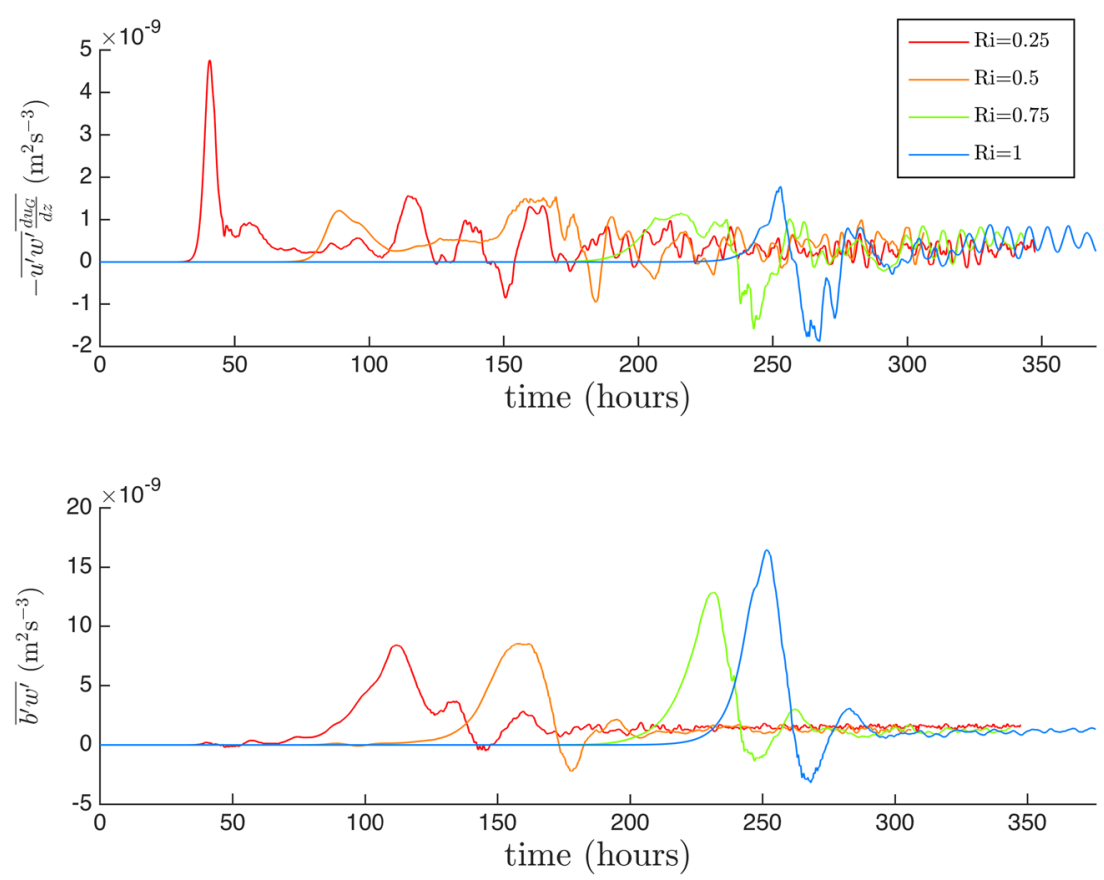
window as large as possible given the simulation duration. The means of these time windows are indicated by closed circles in Fig. 6. The range of values in Figs. 11 and 13a represent minimum and maximum values of each quantity over this interval in $\epsilon$, with the circle indicating the mean of the time integrals for each quantity.

Figure 11 shows the time integrated buoyancy flux and geostrophic shear production, normalised by the magnitude of the kinetic energy associated with the background thermal wind, $\mathrm{KE}_{G}$. As expected, given that symmetric instability takes its energy predominantly from geostrophic shear production we see that simulations with lower $\mathrm{Ri}_{0}$ have higher integrated geostrophic shear production. The time integrated buoyancy flux is nearly the same in all simulations. Interestingly, even for the $\mathrm{Ri}_{0}=0.25$ and $\mathrm{Ri}_{0}=0.5$ simulations, the integrated buoyancy flux is significantly larger than the integrated geostrophic shear production.

\subsection{Dissipation and mixing}

Overall, there is a complex set of energy pathways present in this system, identified schematically in Fig. 12. Initially, via primary instabilities, we observe downscale energy transfer from energy reservoirs on the domain scale, approximately $2.5 \mathrm{~km}$, the tilted isopycnals providing potential energy (PE) and kinetic energy associated with the thermal wind $\left(\mathrm{KE}_{G}\right)$. Symmetric instability (SI) transfers energy primarily via geostrophic shear production from the mean kinetic energy reservoir to symmetric instability cells like those visualised in Fig. 3a. Baroclinic instability (BI) transfers energy from PE to baroclinic eddies through the buoyancy flux. The submesoscale motions associated with symmetric instability, baroclinic instability and mixed modes then transfer energy further downscale to 3D turbulence via the range of secondary instabilities that occur on saturation of the primary instabilities.

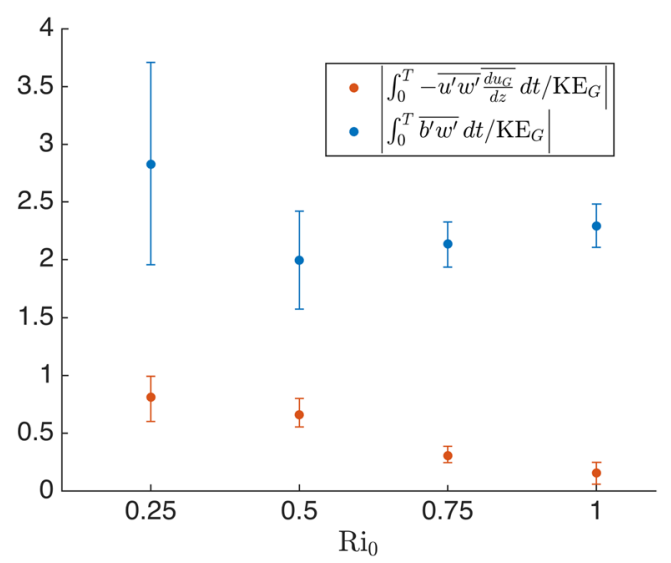

Fig. 11 A comparison between total geostrophic shear production and buoyancy flux for each simulation
Finally, dissipation and mixing provide the two major small-scale energy sinks in the system. Dissipated energy is entirely lost from the system. However, mixing allows some return-flow of energy to one of our large-scale energy reservoirs, the potential energy (PE), as indicated by the dashed line in Fig. 12. Mixing, by decreasing the mean stratification while $M^{2}$ is held fixed, causes the steepening of isopycnals and hence an increase the system's potential energy.

The buoyancy flux, $\overline{w^{\prime} b^{\prime}}$, is associated with a transfer from potential energy to kinetic energy when $\overline{w^{\prime} b^{\prime}}>0$, and mixing where $\overline{w^{\prime} b^{\prime}}<0$. To separate these contributions, we split the buoyancy flux into a large scale component associated with fluxes from the background potential energy to submesoscales by the primary symmetric and baroclinic instability, $\mathcal{B}$, and a small scale component associated with mixing, $\mathcal{M}$,

$B=\mathcal{B}+\mathcal{M}$.

A small-scale cutoff wavenumber $k_{\text {cutoff }}$ is defined as the value of $|\mathbf{k}|=\sqrt{k^{2}+\ell^{2}}$ which minimises

$\operatorname{Re}\left(\int_{|\mathbf{k}|}^{|\mathbf{k}|_{\max }} \tilde{w} \tilde{b}^{*} d\left|\mathbf{k}^{\prime}\right|\right)$.

Instantaneous mixing, $\mathcal{M}$, is then defined as the integral from the wavenumber cutoff, $k_{\text {cutoff, }}$ to the largest wavenumber, $k_{\max }$,

$\mathcal{M}=\operatorname{Re}\left(\int_{0}^{H} \int_{k_{\text {cutoff }}}^{k_{\max }} \tilde{b} \tilde{w}^{*} d k d z\right)$,

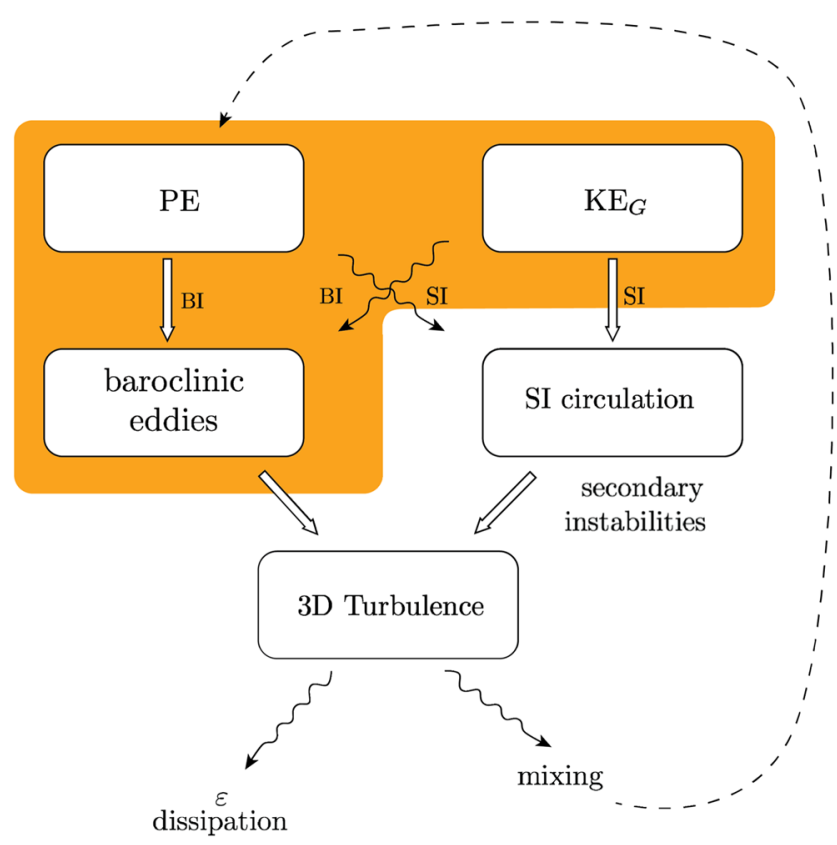

Fig. 12 Schematic illustrating the various energy pathways present in the system. The orange boundary indicates the pathways present in a typical low-resolution simulation where grid-scales would be too small to resolve symmetric or secondary instabilities 
Fig. 13 a Comparison between total energy dissipation and mixing normalised by kinetic energy in the background thermal wind, $\mathrm{KE}_{G}$; b mixing efficiency in terms of flux Richardson number $\mathrm{Ri}_{f}$ for each simulation (a)

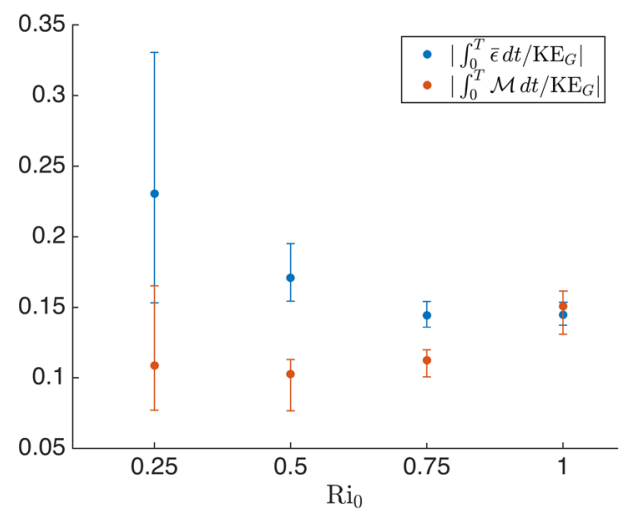

(b)

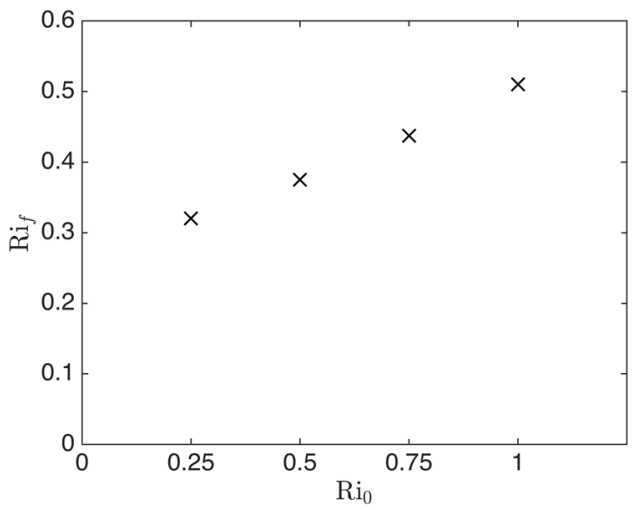

and the large-scale buoyancy flux, $\mathcal{B}$, is defined as the contributions from

$\mathcal{B}=\operatorname{Re}\left(\int_{0}^{H} \int_{0}^{k_{\text {cutoff }}} \tilde{b} \tilde{w}^{*} d k d z\right)$.

Figure 13a shows the total energy lost to dissipation and mixing normalised by the kinetic energy associated with the background thermal wind, $\mathrm{KE}_{G}$, throughout each simulation. Together, mixing and dissipation represent a non-negligible fraction of the initial kinetic energy with dissipation reaching up to around $24 \%$ and mixing, $15 \%$ of $\mathrm{KE}_{G}$.

There is also notable variation with initial Richardson number, with dissipation decreasing with increasing $\mathrm{Ri}_{0}$ and, conversely, mixing increasing with increasing $\mathrm{Ri}_{0}$. These two trends, when measured in terms of a flux Richardson number, $\mathrm{Ri}_{f}=-\mathcal{M} /(\bar{\epsilon}-\mathcal{M})$, which can be interpreted as an approximation to the mixing efficiency, combine to result in a strong positive linear trend, with higher mixing efficiencies occurring with higher $\mathrm{Ri}_{0}$, as shown in Fig. $13 \mathrm{~b}$.

Thus, although less energy is dissipated in simulations with larger $\mathrm{Ri}_{0}$, the larger bulk stratification leads to higher rates of mixing. The trend in $\mathrm{Ri}_{f}$ is reminiscent of that seen at much lower values of Ri (e.g., Deusebio et al. 2015; Linden 1979), although it is important to note that $\mathrm{Ri}_{0}$ is an initial bulk value and not necessarily representative of the Richardson number during individual mixing events.

\subsection{Vertical velocities}

Submesoscales are known to be associated with large vertical velocities (e.g., Capet et al. 2006; Mahadevan and Tandon 2008; Thomas et al. 2008). Figure 14 shows the time evolution of the root mean square vertical velocity, averaged over the full domain. There is some variation in the maximum rms vertical velocity attained in each case, with higher values achieved for higher $\mathrm{Ri}_{0}$ simulations. However, each simulation attains a similar rms vertical velocity of around $2 \mathrm{~cm} \mathrm{~s}^{-1}$ in the latter stages.
In previous frontal simulations by (Mahadevan and Tandon 2006), with a domain of $96 \mathrm{~km} \times 48 \mathrm{~km}$ in the horizontal, with surface wind forcing, but coarser horizontal resolution $(0.5 \mathrm{~km}$ grid spacing $)$, the maximum vertical velocities observed were approximately $1-2 \mathrm{~mm} \mathrm{~s}^{-1}$. Here, we see peaks in rms vertical velocity that are more than an order of magnitude greater. In the $\mathrm{Ri}_{0}=0.25$ simulation, we achieve maximum values as high as $22 \mathrm{~mm} \mathrm{~s}^{-1}$, and in the $\mathrm{Ri}_{0}=1$ case, this maximum value increases to around $30 \mathrm{~mm} \mathrm{~s}^{-1}$.

The first rms velocity maxima seen in the $\mathrm{Ri}_{0}=0.25$ and 0.5 simulations, at 42 and $89 \mathrm{~h}$, respectively, are associated with significant upwelling/downwelling along frontal bands at the top and bottom of the domain, as seen in Fig. $5 b$. Global maxima occur as vertical velocities grow along filament structures seen in the later stages of each simulation as displayed in Fig. 15a. There is substantially larger negative vertical velocities at the surface (and corresponding large positive vertical velocities near the bottom of the domain) consistent with the circulation associated with frontogenesis (Hoskins 1982).

The frontal structure can be examined further by looking at vertical slices across the filaments, as shown in Fig. 15.

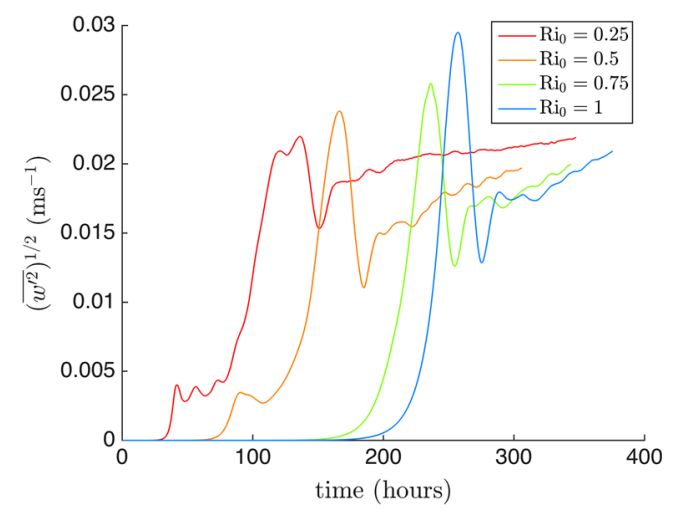

Fig. 14 Temporal evolution of the root mean square vertical velocity for all simulations 


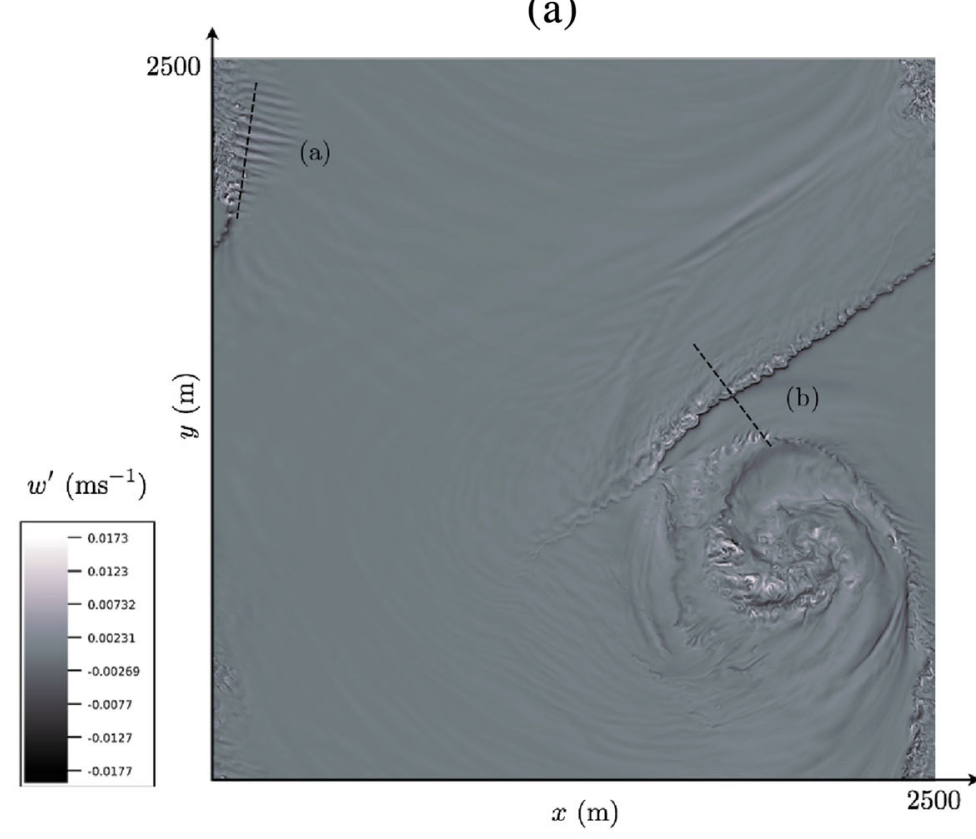

(b)

(c)
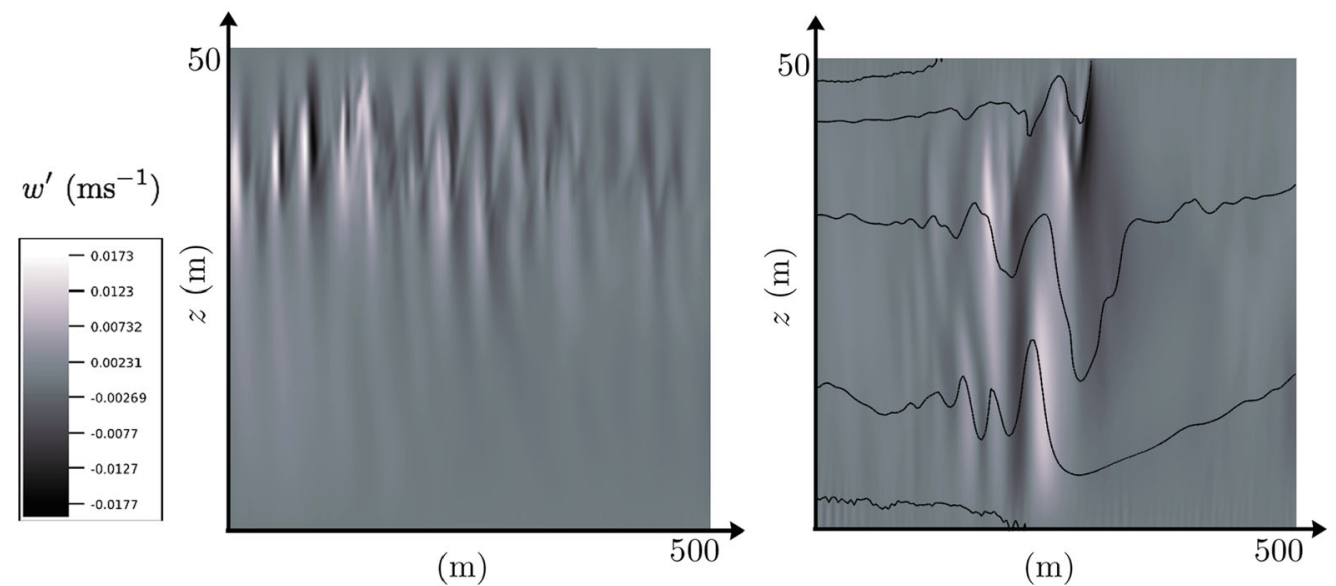

(d)
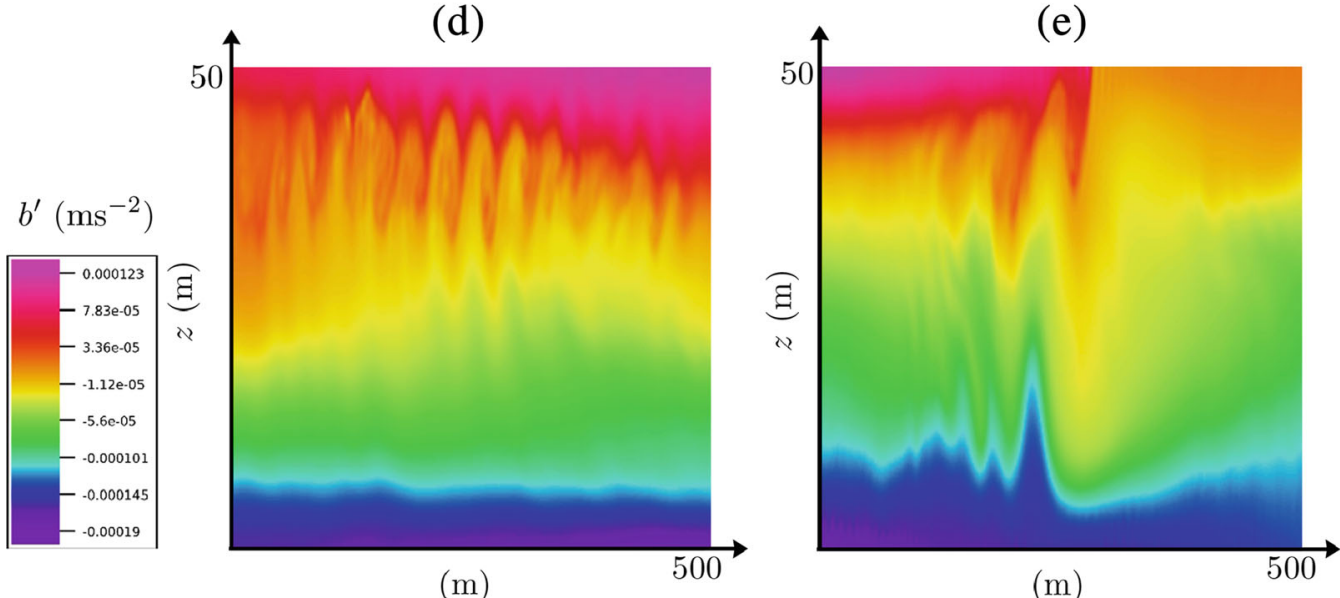

Fig. 15 a A snapshot of the vertical velocity, $w^{\prime}$, at 5-m depth in the $\mathrm{Ri}_{0}=0.25$ simulation at $136 \mathrm{~h}$, the approximate time of the global maxima of $w_{\text {rms }}$ as shown in Fig. 14. Panels $\mathbf{b}$ and $\mathbf{c}$ through the depth,

$z$, of the domain show vertical velocities, $w^{\prime}$, taken at the dashed lines indicated in Fig. 15a. Panels $\mathbf{d}$ and $\mathbf{e}$ are the same slices as above for buoyancy, $b^{\prime}$ 
The flow appears to develop further secondary shear instabilities. For example, Figs. 15b, d shows a vertical slice with Kelvin-Helmholtz billows between 0 and $10 \mathrm{~m}$ depth, at the end of a long filament structure protruding from the eddy in the $\mathrm{Ri}=0.25$ simulation. Figure $15 \mathrm{c}$ provides further evidence for strong frontogenesis along the filaments, where, with black contours indicating isopycnals, we see strong downwelling to the right of the surface front.

\section{Conclusion}

Here, we examined the linear stability and nonlinear evolution of the 'Eady model', a fluid with initially uniform density gradients that is unstable to symmetric and baroclinic instability. A numerical stability analysis showed a smooth transition with increasing balanced Richardson number from the dominance of symmetric instability to that of baroclinic instability, with the additional possibility of a wide range of 3-dimensional 'mixed modes'. Mixed modes, while never quite being the fastest growing, can often have growth rates within just a few percent of the maximum. Mixed modes may draw energy via both geostrophic shear production and buoyancy flux.

In 3-dimensional simulations of an idealised ocean front with $\mathrm{Ri}_{0}<1$, we observed growth of symmetric modes, followed by the development of mixed and baroclinic modes. Here, the early appearance of symmetric instability and mixed mode instabilities, whose growth rates agree well with the linear predictions, led to restratification and, given $M^{2}$ and $f$ were fixed, resulted in an increase in Ri. This transition to a new background state implied that baroclinic modes become fastest growing, dominating the latter stages of each simulation. Despite the flow being nonlinear, the growth rate of the baroclinic modes closely matches predictions made using the mean Richardson number after saturation of symmetric instabilities.

Despite the fact that they are never the most unstable, mixed modes represented the largest contribution to the turbulent kinetic energy for a significant period of time in simulations with $\mathrm{Ri}_{0}<1$. Ultimately, the time integrated buoyancy flux was the dominent energy source for all simulations but time integrated geostrophic shear production represented a sizeable proportion of the kinetic energy (when compared with kinetic energy associated with the thermal wind) in simulations with $\mathrm{Ri}_{0}<1$. This could have important implications for sub-grid scale parametrisations attempting to capture the effects of submesoscale instabilities. For example, the parametrisation of FFH (Fox-Kemper et al. 2008a) attempts to recreate the effects of baroclinic instability by releasing potential energy via adiabatic overturnings but has no mechanism for sub-grid scale kinetic energy release. It therefore does not capture the potentially significant effects of non-baroclinic submesoscale instabilities via sizeable geostrophic shear production.

By capturing symmetric, mixed mode, and baroclinic instabilities together for the first time, the simulations presented here highlight the presence of a number of interesting secondary instability mechanisms, including the transition to Kelvin-Helmholtz instability resulting from growing symmetric instability. In addition, we observed a horizontal shear instability occurring at the edges of growing frontal band structures. Overall, these transitions to secondary instability provide us with a mechanism to transfer from mesoscale features to small scales at which energy can be dissipated and mixed.

Dissipation and mixing in these simulations are significant fractions of the mean kinetic energy of the thermal wind. Trends in dissipation and mixing with increasing initial Richardson number, $\mathrm{Ri}_{0}$, resulted in a monotonically increasing bulk Richardson number, $\mathrm{Ri}_{f}$, suggestive of mixing efficiencies being enhanced as $\mathrm{Ri}_{0}$ increases. Interestingly, there was significant dissipation and mixing even in the case where $\mathrm{Ri}_{0}=1$, i.e., with small scale symmetric and mixed mode instabilities absent. The monotonic mixing efficiency relationship could perhaps even extend beyond $\mathrm{Ri}_{0}=1$, the largest value of $\mathrm{Ri}_{0}$ taken here. These results run counter to the perception that we have a simple competition between surface forcing driving mixing and submesoscales driving restratification, with these results demonstrating that submesoscales in the absence of surface forcing can result in significant dissipation and mixing.

All simulations resulted in enhanced vertical velocity. We found rms vertical velocities an order of magnitude greater than in comparable simulations at lower resolution. Peaks in vertical velocity appeared to be highly localised along fronts, with the sign of the vertical velocity at the surface (and bottom of the domain) at late times highlighting the presence of continued frontogenesis at filament structures protruding from the large late-time eddy structure formed.

The processes identified in this paper, particularly the enhanced mixing and vertical velocities, may have important implications for upper ocean turbulence in the presence of submesoscales. In particular, the large but highly localised vertical velocity could have an important impact on nutrient supply and primary production. A coupling of our dynamic model with a phytoplankton model would be an interesting expansion to further investigate the net effects of these dynamics on a phytoplankton bloom.

In this work, we focused on the Eady model, an idealised setup with linear density and velocity profiles and rigid horizontal boundaries. While the signatures of symmetric and baroclinic instability have both been seen in observations of localised fronts (Thomas et al. 2013; D'Asaro et al. 2011; Thomas and Joyce 2010) the relative importance of the interaction between instabilities remains uncertain. In 
future work, we hope to explore this question in a less idealised configuation. In particular, we hope to examine initial conditions with a more realistic frontal structure and stratification, allowing us to investigate the behaviour of isolated fronts.

Open Access This article is distributed under the terms of the Creative Commons Attribution 4.0 International License (http:// creativecommons.org/licenses/by/4.0/), which permits unrestricted use, distribution, and reproduction in any medium, provided you give appropriate credit to the original author(s) and the source, provide a link to the Creative Commons license, and indicate if changes were made.

\section{Appendix A: Linear stability analysis}

In order to solve Eq. 2, we proceed using a method outlined in (Stone 1970). We first discretise Eq. 2 using second-order central difference formulae giving

$w_{n+1}=\frac{1}{A_{n}}\left(B_{n} w_{n}+C_{n} w_{n-1}\right), \quad n=0, \ldots, N$,

where

$A_{n}=h_{n}\left(1-h_{n}^{2}\right)-k \Delta+i \ell \Delta h_{n}$,

$B_{n}=2 h_{n}\left(1-h_{n}^{2}\right)+\operatorname{Ri}\left(k^{2}+\ell^{2}\right) \Delta^{2} h_{n}+2 i k \ell \Delta^{2}$,

$C_{n}=-h_{n}\left(1-h_{n}^{2}\right)-k \Delta+i \ell \Delta h_{n}$

and

$h_{n}=\sigma+k z_{n}, \quad w_{n}=w\left(z_{n}\right), \quad z_{n}=n \Delta-H, \quad \Delta=\frac{1}{N+1}$.

Imposing the no-flux boundary condition at the bottom gives $w_{0}=0$ and we set $w_{1}=1$, an arbitrary choice taken to fix the undetermined amplitude of the eigenfunction. Note that Stone instead discretised an analytically simplified version of (2), equation 2.6 from Stone (1970). We instead began with Eady's original form, Eq. 2, as we found this more numerically stable using the described method.

To proceed we require an initial guess for $\sigma$ to initialise the chosen iterative shooting method. For each Ri, we have, for values of $k$ and $\ell$ asymptotically close to the symmetric and baroclinic axes, analytic expressions for the growth rate. Close to the symmetric axis, with $k \ll 1$, equation (2.7) from Stone (1970) can be used to give an initial estimate for $\sigma$ while, close to the baroclinic axis, with $\ell \ll 1$, we can use equation (4.4) from Stone (1970).

The shooting method uses these initial guesses as follows. For a given Ri, we plug the initial guess for $\sigma$ at the chosen $k$ and $\ell$ into Eq. A.5, iterating the recurrence relation in $n$ until we find a solution at the top of the domain, $w_{N}$. Next, we check how close we are to satisfying the upper, no-flux boundary condition $w_{N}=0$. If our first guess for $\sigma$ does not satisfy $w_{N}<\epsilon$ for $\epsilon \ll 1$, then we proceed using linear extrapolation as follows. Adding a small parameter $\epsilon$ with $\epsilon \ll 1$ to our initial guess for $\sigma$, we compute a second value of $w_{N}$. Making the assumption that there is a linear variation of $w_{N}$ with $\sigma$, i.e., that $w=m \sigma+c$ for some $m, c \in \mathbb{R}$, we use the two values we have for $w_{N}$ and $\sigma$, the initial solution and that computed with $\epsilon$ added, to calculate $m$ and $c$, giving a new, third value of $w_{N}$ and $\sigma$. We iterate using this procedure with our last two computed values of $w_{N}$ and $\sigma$ at each stage until we have either converged on a solution satisfying the upper boundary condition, i.e. requiring that $w_{N}<\epsilon$ with $\epsilon \ll 1$, or have reached the maximum cutoff number of iterations permitted, warning us that the chosen method is non-convergent (here we found a maximum of 10 iterations sufficient).

For values of $k$ and $\ell$ further from the symmetric and baroclinic axis $(\ell \gg 0, k \gg 0)$, where analytic expressions are not available, we must use an alternative method to inform an initial guess for $\sigma$. We found that using previously computed values of $\sigma$ nearby in $(k, \ell)$ space was helpful. Beginning at $(k=0, \ell=0)$ and moving right and upwards throughout $(k, \ell)$ space, we found that the optimum method for choosing an initial guess at each new point, i.e., that which converged upon a solution most often, was to take the smallest computed value of $\sigma$ found at the corresponding nearest left, lower and lower diagonal points already computed.

\section{References}

Arobone E, Sarkar S (2015) Effects of three-dimensionality on instability and turbulence in a frontal zone. J Fluid Mech 784:252-273

Boccaletti G, Ferrari R, Fox-Kemper B (2006) Mixed layer instability and restratification. J Phys Oceanogr:37

Capet X, McWilliams C, Molemaker MJ, Shchepetkin AF (2008) Mesoscale to Submesoscale Transition in the California Current System. Part I Flow Structure, Eddy Flux and Observational Tests. J Phys Oceanogr:38

Cushman-Roisin B, Beckers J (2011) Introduction to Geophysical Fluid Dynamics: Physical and Numerical Aspects

D'Asaro E, Lee C, Rainville L, Harcourt R, Thomas L (2011) Enhanced turbulence and energy dissipation at ocean fronts. Science 332:318-322

Deusebio E, Caulfield CP, Taylor JR (2015) The intermittency boundary in stratified plane Couette flow. J Fluid Mech 781:298-329

Eady ET (1949) Long waves and cyclone waves. Tellus 1: $33-52$

Fox-Kemper B, Ferrari R, Hallberg R (2008a) Parameterization of mixed layer eddies. part i: theory and diagnosis. J Phys Oceanogr 38

Fox-Kemper B, Ferrari R, Hallberg R (2008b) Parameterization of mixed layer eddies. part i: theory and diagnosis. J Phys Oceanogr 38(6):1145-1165

Haine TWN, Marshall J (1997) Gravitational symmetric, and baroclinic instability of the ocean mixed layer. J Phys Oceanogr:28

Hoskins BJ (1974) The role of potential vorticity in symmetric stability and instability. Q J R Meteorol Soc 100:480-482 
Hoskins BJ (1982) The Mathematical Theory of Frontogenesis. Annu Rev Fluid Mech 14:131-151

Joyce TM, Thomas LN, Bahr F (2009) Wintertime observations of subtropical mode water formation within the gulf stream. Geophys Res Lett 36(2)

Lévy M, Ferrari R, Franks PJS, Martin AP, Rivière P (2012) Bringing physics to life at the submesoscale. Geophys Res Lett 39:L14602.10.1029/2012GL052756

Linden PF (1979) Mixing in stratified fluids. Geophys Astrophys Fluid Dyn 13:3-23

Mahadevan A, Tandon A (2006) An analysis of mechanisms for submesoscale vertical motion at ocean fronts. Ocean Model:14

McWilliams JC, Molemaker MJ, Yavneh I (2004) Ageostrophic, anticyclonic instability of a geostrophic, barotropic boundary current. Phys Fluids (1994-present) 16(10):3720-3725

Molemaker MJ, McWilliams JC, Capet X (2010) Balanced and unbalanced routes to dissipation in an equilibrated eady flow. J Fluid Mech 654:35-63

Rudnick DL, Ferrari R (1999) Compensation of horizontal temperature and salinity gradients in the ocean mixed layer. Science 283(5401):526-529

Shakespeare CJ, Taylor JR (2013) A generalized mathematical model of geostrophic adjustment and frontogenesis: Uniform potential vorticity. J Fluid Mech 736:366-413
Stone PH (1966) On non-geostrophic baroclinic stability. J Atmos Sci 23:390-400

Stone PH (1970) On non-geostrophic baroclinic stability: Part II. J Atmos Sci 27:721-726

Stone PH (1972) On non-geostrophic baroclinic stability: Part III. The momentum and heat transports. J Atmos Sci 29:419426

Taylor JR (2008) Numerical simulations of the stratified oceanic bottom boundary later. $\mathrm{PhD}$ thesis University of California

Taylor JR, Ferrari R (2009) On the equilibrium of a symmetrically unstable front via a secondary shear instability. J Fluid Mech 622:103-113

Taylor JR, Ferrari R (2011) Ocean fronts trigger high latitude phytoplankton blooms. Geophys Res Lett 38(23)

Thomas LN, Joyce TM (2010) Subduction on the northern and southern flanks of the gulf stream. J Phys Oceanogr 40:429438

Thomas LN, Tandon A, Mahadevan A (2008) Submesoscale Processes and Dynamics. AGU geophysical monograph series ocean modeling in an Eddying Regime: 177

Thomas LN, Taylor JR, Ferrari R, Joyce TM (2013) Symmetric instability in the gulf stream. Deep-Sea Res II 91:96-100

Vanneste J (1993) The Kelvin-Helmholtz instability in a nongeostrophic baroclinic unstable flow. Math Comput Model 17(1):149-154 